\title{
Safety of escitalopram in pregnancy: a case series
}

\author{
This article was published in the following Dove Press journal: \\ Neuropsychiatric Disease and Treatment \\ 7 September 2013 \\ Number of times this article has been viewed
}

\section{Cesario Bellantuono \\ Francesca Bozzi \\ Laura Orsolini}

Psychiatric Unit and DEGRA Center, United Hospital and Academic

Department of Experimental and Clinical Medicine, Polytechnic University of Marche, Ancona, Italy
Correspondence: Laura Orsolini Psychiatric Unit and DEGRA Center, United Hospital and Academic Department of Experimental and Clinical Medicine, Polytechnic University of Marche, Via Conca 7I, 60020 Ancona, Italy

Tel +3907 I5963308

$\mathrm{Fax}+3907$ I5963313

Email laura.orsolini@hotmail.it
Background: The aim of this paper is to report maternal and neonatal outcomes in pregnant women treated with escitalopram during pregnancy and breastfeeding.

Methods: Women enrolled in the DEGRA Database at the Clinic of Affective Disorders in Pregnancy and Postpartum in Italy, treated during pregnancy with escitalopram and followed up throughout pregnancy, were included in this study. All patients provided written informed consent and the study was approved by the local ethics committee. Psychiatric diagnoses were assessed using the Structured Clinical Interview for DSM-IV (Diagnostic and Statistical Manual of Mental Disorders - Fourth Edition) Axis I Disorders and symptoms were assessed using the Hamilton Rating Scale for Depression (17 items) and Hamilton Rating Scale for Anxiety (14 items). Clinical and sociodemographic characteristics as well as maternal and neonatal outcomes were recorded.

Results: The case histories of seven pregnant women treated for depression and/or anxiety disorders with escitalopram were reported. Four women were also treated with benzodiazepines All pregnancies were full-term and all newborns had normal Apgar scores. There were no major malformations or miscarriages following exposure to escitalopram. Mild withdrawal syndrome was reported only in a newborn who was also exposed to a benzodiazepine. Two infants exposed to escitalopram during breastfeeding did not show any health problems.

Conclusion: Our experience with use of escitalopram in pregnant women did not reveal any maternal or neonatal concerns. However, considering the few cases analyzed and the paucity of published literature, no conclusions can be drawn on its safety profile in pregnancy and breastfeeding.

Keywords: escitalopram, pregnancy, breastfeeding, major malformations, perinatal complications

\section{Introduction}

Major depressive disorder, in many cases in comorbidity with anxiety disorders, frequently occurs during pregnancy. Approximately $10 \%-15 \%$ of pregnant women experience these disorders, ${ }^{1,2}$ with clinically relevant consequences for the outcome of pregnancy. In fact, depressed women do not seek early prenatal care and are more likely to adopt other unsafe behaviors, such as inadequate diet and use of tobacco, alcohol, and other harmful substances. Further, women with untreated major depressive disorder can be at risk of self-harm or suicide. ${ }^{3}$ High rates of relapse were reported by women with depression who discontinued antidepressant therapy at the time of conception, compared with women who continued therapy throughout the pregnancy, in a study conducted by Cohen et al. ${ }^{4}$ There is an unwillingness among physicians and patients to use antidepressant drugs during the gestational period, 
because there are conflicting results about the use of such drugs, and the risk of major congenital malformations. ${ }^{5-8}$ The Canadian Paediatric Society recommends that satisfactory treatment of depression in pregnancy is important for the well-being of both the mother and infant, and that individual risk-benefit assessment must be made when a selective serotonin reuptake inhibitor needs to be prescribed during pregnancy. ${ }^{9}$

Nowadays, the selective serotonin reuptake inhibitor, escitalopram, is considered to be one of the most effective and well tolerated agents for the treatment of moderate and severe major depressive disorder, ${ }^{10}$ as well as for the treatment of anxiety disorders. Overall, the data available on its use during pregnancy and breastfeeding suggest a good safety profile. ${ }^{5}$ The aim of this paper is to report the maternal and neonatal outcomes of seven pregnant women treated with escitalopram during their pregnancy.

\section{Materials and methods}

Cases of women who were treated during pregnancy with escitalopram were drawn from a recently implemented database (www.degradatabase.it, September 2009). The DEGRA Database now includes 110 pregnant women who have been followed during their pregnancy at the Clinic of Affective Disorders in Pregnancy and Postpartum (DEGRA Center), Academic Psychiatric Unit, United Hospitals of Ancona, Italy. The psychiatric diagnosis of patients at their first visit was made using the Structured Clinical Interview for DSM-IV (Diagnostic and Statistical Manual - Fourth Edition) Axis I Disorders, ${ }^{11}$ while severity was assessed using the Hamilton Rating Scale for Depression (HAM-D-17 items) and Hamilton Rating Scale for Anxiety (HAM-A-14 items). All patients were fully informed about the benefits, risks, and potential adverse effects of treatment with the drug during pregnancy and breastfeeding, as well as on the risks of untreated depression/ anxiety disorder in pregnancy, by a senior psychiatrist (CB) before initiating treatment. Pregnant women were also asked to sign a consent form approved by the ethics committee.

Only pregnant women treated with escitalopram and followed up by our team throughout pregnancy and, in some cases, also during postpartum, were included in this study. Clinical and sociodemographic characteristics as well as maternal and neonatal outcomes were recorded during the study period. Neonatal outcomes were assessed in terms of mean birth weight, routine pediatric examination, Apgar scores, and examination for major congenital malformations or other minor anomalies.

\section{Results}

We report seven cases of women treated with escitalopram during their pregnancy drawn from the DEGRA Database. Clinical and sociodemographic characteristics and neonatal outcomes of these seven cases are summarized in Table 1. Mean age ( \pm standard deviation) at the time of presentation was $34.14 \pm 3.76$ (range 29-38) years. All women were of Caucasian ancestry, living with their partners, and currently working. For four women, this was their first pregnancy; three women had previously given birth to healthy babies after full-term pregnancies. Two patients were diagnosed with panic disorder, one with generalized anxiety disorder, and four with major depressive disorder (one of these also had panic disorder). Four patients were moderate smokers (5-10 cigarettes/day).

Six women were exposed to escitalopram (10-20 mg/day) at conception; of these, two received escitalopram throughout their pregnancy, one discontinued treatment at week 8 and restarted at week 24 (up to delivery) due to a new severe episode of anxiety, and one was switched to paroxetine because of lack of efficacy of escitalopram at week 16 . Two women decided to discontinue treatment at week 8 and 13, respectively. Only one patient was treated with escitalopram from week 21 to delivery. All cases but one showed a good clinical response to escitalopram.

Four patients were also taking low doses of alprazolam, lorazepam, and clonazepam at bedtime. One patient, affected by thyroid disease, was exposed throughout pregnancy to L-thyroxine.

All pregnancies were full-term. The mean birth weight of the newborns was $2,870 \pm 269.01 \mathrm{~g}$ (range 2,400-3,100 g), which is considered to be in the normal range (2,500-4,500 g); only one infant showed a low birth weight of 2,400 g. All newborns had normal Apgar scores at 1 and 5 minutes. We did not detect any major congenital malformations or other minor anomalies in the newborns.

One newborn also exposed to paroxetine (20-40 mg/day) and a benzodiazepine during late pregnancy developed a withdrawal reaction characterized by hypoglycemia (controlled with dextrose administration), lethargy, hypotonia, decreased primitive reflexes, and poor responsiveness to noxious stimuli. Symptoms improved within 48 hours and the newborn did not require any other medical intervention.

During the postpartum period, all women and newborns were followed up for 6 months. Only two patients decided to continue drug treatment with escitalopram ( $10 \mathrm{mg} /$ day $)$ until 1 and 5 months after delivery, respectively, in order to prevent 


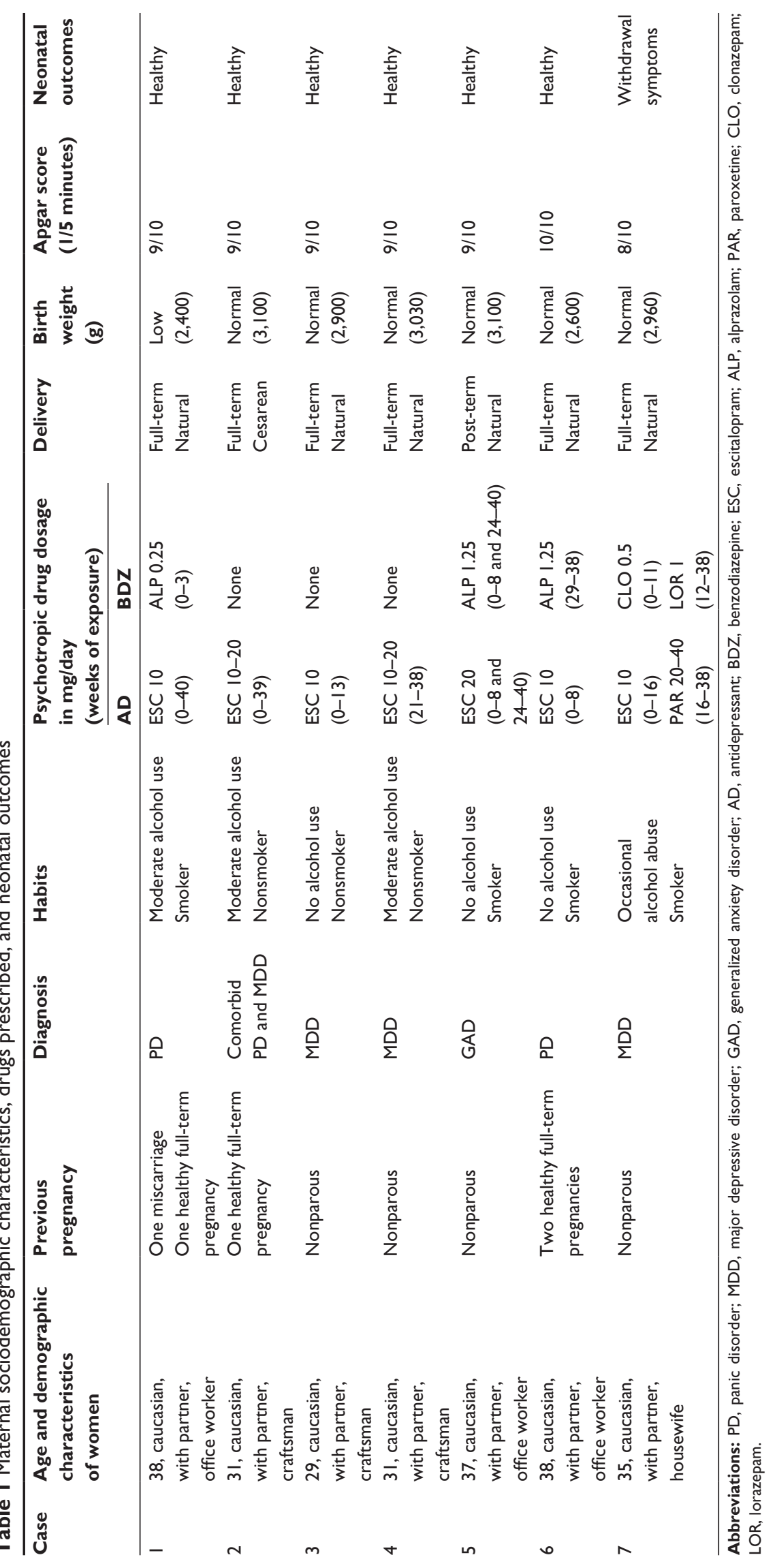


postpartum depression. All infants exposed to escitalopram showed no health problems during the follow-up period.

\section{Discussion}

The safety profile of escitalopram during the gestational period is an important topic for the health care community because the prescription of such drugs during pregnancy and in women of childbearing age affected by psychiatric illnesses is still up for debate. There is still some uncertainty about the relative risk concerning the use of escitalopram in pregnancy, as data on its safety are available only after the drug has been prescribed for many years. On the other hand, it is well documented that untreated maternal major depressive disorder and/or anxiety disorders, as well as other severe psychiatric illnesses, can have disruptive consequences on the outcome of pregnancy and on the safety of the newborn. ${ }^{3,49}$ In our case series, no major malformations were detected after exposure to escitalopram during early pregnancy. This is consistent with the available literature suggesting that escitalopram is not associated with an increased risk of major or minor abnormalities. ${ }^{5,7,12}$ We reported only one underweight newborn, a finding which is consistent with other evidence showing an increased risk of low birth weight of newborns exposed in utero to escitalopram. ${ }^{7}$ We also observed a case of withdrawal syndrome, even though the event occurred in a newborn whose exposure to escitalopram was stopped at 16 weeks but who was also exposed to paroxetine and clonazepam later during the pregnancy. Therefore, it is unlikely that escitalopram was responsible for this adverse effect, as this drug is not frequently associated with withdrawal reactions at therapeutic doses. ${ }^{13}$

This case series demonstrates the need for antidepressant therapy during pregnancy and provides valuable information on the use of escitalopram in pregnancy in a real-life clinical setting, with a range of depression-related diagnoses and exposure to escitalopram, concomitant use of other drugs, and in the presence of other risk factors. However, results from this case series should be interpreted with caution due to the low number of cases reported and the lack of controls. Due to these limitations, the causation, association, strength of association, and effect of confounders on the findings, eg, depression, maternal smoking, and socioeconomic status, are uncertain.

Our findings are in agreement with evidence from large population-base studies reported in the literature, and provide data to support the safety of escitalopram in pregnancy in terms of no increased risk of major malformations. ${ }^{6,7,12}$ However, randomized controlled studies with a larger sample size are required in order to establish the safety profile of escitalopram in pregnancy and in breastfeeding women.

A range of depression rating scales are available for assessment of symptom severity. We used the HAM-D-17 and HAM-A-14 scales to assess symptoms of depression and anxiety during the follow-up period. However, although beyond the scope of this study, other scales, such as the Montgomery-Åsberg Depression Rating Scale, may better differentiate biological and somatic symptoms of depression from symptoms commonly experienced during pregnancy.

\section{Conclusion}

The data from this small preliminary case series, together with the existing evidence, suggest that escitalopram does not appear to be associated with a higher risk of adverse maternal and neonatal outcomes when used by women during the gestational period, despite the fact that perinatal complications with other selective serotonin reuptake inhibitors have been reported in the literature. Given the paucity of data on the safety of escitalopram in pregnancy and the risk involved with nontreatment of major depressive disorder during pregnancy, any additional data are welcomed. However, considering the low number of cases and lack of controls in this series, it is prudent not to draw definitive conclusions on its safety during pregnancy and breastfeeding. These preliminary data are from a recently implemented database; therefore, future findings with larger patient numbers will add to the literature on the treatment of depression during pregnancy and the safety of escitalopram in the newborn.

\section{Author contributions}

$\mathrm{CB}$, the senior psychiatrist responsible for treatment of patients afferent to the Clinic of Affective Disorders in Pregnancy and Postpartum, designed and planned the research. FB and LO performed the literature search and database analysis. All authors participated in drafting the manuscript and approving the final version for submission. The authors thank David Bellantuono for developing the online DEGRA Database used in this study.

\section{Disclosure}

Editorial assistance was provided by Raelene Simpson on behalf of inScience Communications, Springer Healthcare. This assistance was supported by Lundbeck. Otherwise, there are no potential conflicts of interest to disclose and no commercial support or funding was received for the study. 


\section{References}

1. Birndorf CA, Madden A, Portera L, Leon AC. Psychiatric symptoms, functional impairment, and receptivity toward mental health treatment among obstetrical patients. Int J Psychiatry Med. 2001;31(4): $355-365$.

2. Grant BF, Goldstein RB, Chou SP, et al. Sociodemographic and psychopathologic predictors of first incidence of DSM-IV substance use, mood and anxiety disorders: results from the Wave 2 National Epidemiologic Survey on Alcohol and Related Conditions. Mol Psychiatry. 2009;14(11):1051-1066.

3. Yonkers KA, Wisner KL, Stewart DE, et al. The management of depression during pregnancy: a report from the American Psychiatric Association and the American College of Obstetricians and Gynecologists. Gen Hosp Psychiatry. 2009;31(5):403-413.

4. Cohen LS, Altshuler LL, Harlow BL, et al. Relapse of major depression during pregnancy in women who maintain or discontinue antidepressant treatment. JAMA. 2006;295(5):499-507.

5. Bellantuono C, Bozzi F, Orsolini L, Catena-Dell'Osso M. The safety of escitalopram during pregnancy and breastfeeding: a comprehensive review. Hum Psychopharmacol. 2012;27(6):534-539.

6. Einarson A, Choi J, Einarson TR, Koren G. Incidence of major malformations in infants following antidepressant exposure in pregnancy: results of a large prospective cohort study. Can J Psychiatry. 2009;54(4): $242-246$.
7. Klieger-Grossmann C, Weitzner B, Panchaud A, et al. Pregnancy outcomes following use of escitalopram: a prospective comparative cohort study. J Clin Pharmacol. 2012;52(5):766-770.

8. Diav-Citrin O, Ornoy A. Selective serotonin reuptake inhibitors in human pregnancy: to treat or not to treat? Obstet Gynecol Int. 2012; 2012:698947.

9. Jefferies AL; Canadian Paediatric Society, and Fetus and Newborn Committee. Selective serotonin reuptake inhibitors in pregnancy and infant outcomes. Paediatric Child Health. 2011;16(9):562-563.

10. Cipriani A, Furukawa TA, Salanti G, et al. Comparative efficacy and acceptability of 12 new-generation antidepressants: a multipletreatments meta-analysis. Lancet. 2009;373(9665):746-758.

11. First MB, Spitzer RL, Gibbon M, Williams JBW. Structured Clinical Interview for DSM-IV Axis I Disorders, Clinician Version (SCID-1-CV). New York, NY: Biometrics Research Department, New York State Psychiatric Institute, Department of Psychiatry, Columbia University; 1995.

12. Kallen BA, Otterblad OP. Maternal use of selective serotonin re-uptake inhibitors in early pregnancy and infact congenital malformations. Birth Defects Res A Clin Mol Teratol. 2007;79(4):301-308.

13. Tixier H, Feyeux C, Girod S, et al. Acute voluntary intoxication with selective serotonin reuptake inhibitors during the third trimester of pregnancy: therapeutic management of mother and fetus. Am J Obstet Gynecol. 2008;199(5):e9-e12.
Neuropsychiatric Disease and Treatment

\section{Publish your work in this journal}

Neuropsychiatric Disease and Treatment is an international, peerreviewed journal of clinical therapeutics and pharmacology focusing on concise rapid reporting of clinical or pre-clinical studies on a range of neuropsychiatric and neurological disorders. This journal is indexed on PubMed Central, the 'PsycINFO' database and CAS.

\section{Dovepress}

The manuscript management system is completely online and includes a very quick and fair peer-review system, which is all easy to use. Visit http://www.dovepress.com/testimonials.php to read real quotes from published authors. 